\title{
Attentional selection: A salience-based competition for representation
}

\author{
JEFFREY R. W. MOUNTS \\ State University of New York, Geneseo, New York
}

\begin{abstract}
The role of salience in localized attentional interference (LAI) was examined. In two experiments, target discrimination performance was measured as a function of the spatial separation between the target and a salient distractor item. In Experiment 1, both the salience of the distractor and that of a target were manipulated. Distractor salience was manipulated via size changes to the distractor, and target salience was manipulated by using unmasked or onset targets. When the target was of low salience, the magnitude of interference from the distractor increased with distractor salience. However, when the target had an abrupt onset, the distractor had no impact on target performance. In Experiment 2 , the attentional salience of the distractor was manipulated using a probability manipulation. Displays contained both a target and a color singleton distractor. The color singleton produced LAI when it was predictive of the target location but not when it was unpredictive of the target location. The results of both experiments are consistent with models of competition-based attentional selection.
\end{abstract}

Several recent models of visual selective attention propose that attentional selection of an object or region results from a competition among objects for representation within the visual system. These include the model of Deco and Rolls (2004), Cave's (2001) feature gate model, Desimone and Duncan's (1995) biased competition model, and Tsotsos's (e.g., Tsotsos et al., 1995) selective tuning model. These models propose that objects compete for control of visually selective neurons and that the response of a given neuron will represent the properties of only the "winning" object.

Given the architecture of the primate visual system, this competition between two objects should depend on their relative locations in the visual field, among other things. According to Desimone and Duncan's (1995) biased competition model of attention, for example, objects compete with one another for representation at various levels within the visual system. Desimone (1998) points out that this competition should be strongest among objects competing for representation by the same populations of neurons. In general, the proportion of receptive fields shared by a pair of objects will increase with increased spatial proximity; therefore, the degree of competition between objects should increase with decreased spatial separation. Likewise, in the model of Deco and Rolls (2004), Tsotsos et al.'s (1995) selective tuning model, and Cave's (2001) feature gate model, all of which employ a space-based hierarchical pyramid architecture, the extent to which two objects can be processed before coming into competition with one another depends on their spatial separation. Ob-

This work was supported by Grant EY1368701 from the National Eye Institute. Thanks to Cara Milburn for help in data collection and analysis. Correspondence concerning this article may be addressed to J. R. W. Mounts, Department of Psychology, SUNY-Geneseo, Geneseo, NY 14454 (e-mail: mounts@geneseo.edu). jects with greater spatial separation are processed in parallel through higher levels in the hierarchy before coming into competition with one another.

Several recent behavioral studies have provided evidence of such a spatially dependent competition among objects. For example, shape discriminations (see, e.g., Mounts, 2000a, 2000b; Mounts \& Tomaselli, 2005; Turatto \& Galfano, 2001) and line length judgments (Caputo \& Guerra, 1998) have been shown to be slower and less accurate when target stimuli appear near attentionally salient distractors such as color singletons. When observers are asked to identify two targets, their accuracy in identifying both targets improves with increased separation between the targets (see, e.g., Bahcall \& Kowler, 1999). Similarly, when observers are asked to make samedifferent judgments, they become faster and more accurate with increased separation between the two targets (Cutzu \& Tsotsos, 2003; McCarley, Mounts, \& Kramer, 2004). All of these studies suggest that competition between attentionally salient objects occurs when they are spatially proximal and that this competition declines with increased spatial separation. This localized attentional interference (LAI) is consistent with the notions that spatially proximal stimuli may be competing for common pools of neural resources and that increasing the spatial separation between objects allows them to be represented by increasingly independent populations of neurons. Recent fMRI data lend additional support to this claim. For example, Kastner et al. (2001) measured sensory suppression, which is believed to index competition among visual objects. They found that the stimulus separations producing sensory suppression in their experiment varied with visual area, and that these separations yielded good matches with estimates of receptive field sizes of neurons within the respective areas. 
Thus, there is growing support for the notion of competition for representation among objects and for the idea that this competition is spatially mediated, in accordance with the predictions of models of attentional selection such as the feature gate, biased competition, and selective tuning models. In all of these models, the competition among objects is presumed to be rooted in the salience of the competing objects (see, e.g., Reynolds \& Desimone, 2003). This salience can either arise from stimulus-driven factors, such as luminance or feature contrast, or be modulated by more cognitive factors, reflecting top-down attentional biases or attentional set. Indeed, several behavioral studies have shown that LAI can arise with purely top-down selection of the items (e.g., Bahcall \& Kowler, 1999; Mounts \& Gavett, 2004). The purpose of the present study is to test the assumptions of these models regarding the role of salience by evaluating the impact of salience on the magnitude of LAI. ${ }^{1}$

Reynolds, Chelazzi, and Desimone (1999) found neurophysiological support for the importance of attentional salience in competition for selection. When two objects were presented within the receptive field of a V2 or V4 neuron and attention was directed to a location outside the receptive field, the response rate assumed by the neuron was intermediate to that produced by either object alone. In a subsequent experiment, the attentional salience of one of the objects was increased by cuing the monkey to attend to one of the two objects. Attending to one of the stimuli caused the response of the neuron to move toward the level associated with the attended object. Reynolds and Desimone (2003) found similar effects when they manipulated the relative contrast of two stimuli falling within the receptive field of V4 neurons. When attention was directed elsewhere in the visual field, the response rate of the neuron was drawn toward that of the stimulus with the higher contrast. Moreover, they found that attentional salience and physical salience interacted, since attention directed to the stimulus with the lower contrast reduced this effect. They argue that attention acts to increase the effective contrast of a stimulus, allowing it to compete more strongly for neural representation.

There has also been some evidence that attentional salience may modulate the magnitude of LAI in behavioral studies. The results of Experiment 1 of Mounts (2000a) suggest a role of attentional salience in modulating LAI. In that study, a color singleton was used as a distractor item. When the target itself was a form singleton, observers could adopt a singleton search strategy, rendering the color singleton salient. In this condition, LAI was observed surrounding the color singleton distractor. In another condition, the target was not a form singleton and observers had to adopt a feature search strategy. Under this search set, the color singleton should not have had high attentional salience and no LAI was observed surrounding it.

Mounts and Gavett (2004) recently measured the impact of salience on LAI more systematically. They used a compound task (see, e.g., Sperling \& Dosher, 1986) in which observers were cued to monitor two locations for a target that was presented at one of the locations (the other location contained a perceptually similar distractor). The cues used were either size or color changes to placeholder stimuli, with the magnitude of change used to manipulate the relative attentional salience of the two cued items. Data showed that target identification performance improved with spatial separation between the cued locations and that the magnitude of the interference was dependent on the relative attentional salience of the cues. Less interference obtained at the location of the more salient cue, and more interference obtained at the location of the less salient cue. In a subsequent experiment, the same pattern was observed when the salience of the targets was manipulated via the luminance contrast of the targets. In the present study, an attempt is made to extend these findings by replicating them in a search style task, where targets compete for selection with a salient, irrelevant distractor item (see Caputo \& Guerra, 1998; Mounts, 2000a, 2000b; Turatto \& Galfano, 2001).

\section{EXPERIMENT 1}

In this experiment, the salience levels of both the target and the distractor items were manipulated. The target could either appear with an abrupt onset (high salience) or be revealed by unmasking a placeholder item (low salience). Abrupt onsets have been shown to be particularly powerful in capturing attention (see Yantis, 1996, for a review), suggesting that they compete strongly for attentional selection. For example, Yantis and Jonides (1984) found that in a search task, when the target had an abrupt onset, target detection reaction times (RTs) did not vary as a function of the number of distractors, suggesting that the abrupt onsets had a higher attentional priority than the nononset distractors. Moreover, Yantis and Hillstrom (1994) found that it was the appearance of a new object, and not the accompanying luminance increment, that lent the abrupt onsets their higher attentional priority. From the perspective of salience-based competition, Keysers and Perrett (2002) suggest that this competitive advantage for onsets may arise because the neural response to a new stimulus is initially strong and declines over time.

In the present task, the target item was embedded within a display containing multiple distractor items. Prior to the revelation of the target (by onset or unmasking), one of the distractor items underwent a small (low-salience) or large (high-salience) increase in size. We will term this the salient distractor to differentiate it from the other distractors that were included in the display to equate sensory masking across conditions. Franconeri and Simons (2003) found that such size increases also lead to attentional capture, as indexed by search performance. They argue that the increased attentional priority for items undergoing a size increase results because such size increases simulate looming stimuli, which are typically associated with high behavioral urgency. Mounts and Gavett (2004) found that the magnitude of the size manipulations could be used to manipulate the relative attentional salience of stimuli in similar displays. In the present task, if the target and 
the salient distractor are pitted against each other in a salience-based competition for representation, then the observed LAI for target identifications should be sensitive to the salience of the target as well as to that of the distractor, with more salient items being at a competitive advantage. ${ }^{2}$ Specifically, as the salience of the distractor is increased, LAI arising from the distractor should increase; conversely, as target salience is increased, LAI arising from the distractor should decrease.

\section{Method}

Subjects. Twenty-three students from the State University of New York at Geneseo participated in the experiment in exchange for course credit. All of the subjects reported normal or correctedto-normal visual acuity.

Apparatus and Stimuli. Stimuli were presented on a 17-in. Sony G220 monitor set to a resolution of $832 \times 624$ pixels with a frame rate of $75 \mathrm{~Hz}$. The observers were seated $57 \mathrm{~cm}$ from the display, with head stabilization aided through the use of a chin and forehead rest. Responses were made using a PsyScope button box
(New Micros, Dallas). All sessions were conducted in a darkened booth.

Stimulus items were circles with crosses in them. Each stimulus circle subtended $1.85^{\circ}$, and the individual line segments used to create the stimuli were $0.15^{\circ}$ in width. The stimuli were gray (luminance $=2.61 \mathrm{~cd} / \mathrm{m}^{2}$ ) presented against a gray (luminance $=$ $0.28 \mathrm{~cd} / \mathrm{m}^{2}$ ) background. On each trial, a target item was created by removing the left or the right half of the horizontal portion of a cross (yielding a $\mathrm{T}$ with the stem pointing left or right). The subjects made a speeded discrimination response regarding the shape of the target (stem to the left or to the right).

As is shown in Figure 1, stimulus items were presented on one of two imaginary concentric rings with radii of $4.0^{\circ}$ and $6.5^{\circ}$. Trials in which the target was unmasked began with eight equally spaced items on the inner ring. For trials in which the target had an abrupt onset, four items were initially presented on the inner ring, spaced with angular separations of $90^{\circ}$. Both trial types began with eight items equally spaced along the outer ring. Figure 1 shows the sequence and timing of stimulus frames for each type of target. Both sequences included a preview display $(500 \mathrm{msec})$, a frame in which the size of one of the distractor items increased $(52 \mathrm{msec})$, and, finally, the target frame $(160 \mathrm{msec})$. Note that the target item was al-

Unmasked Target
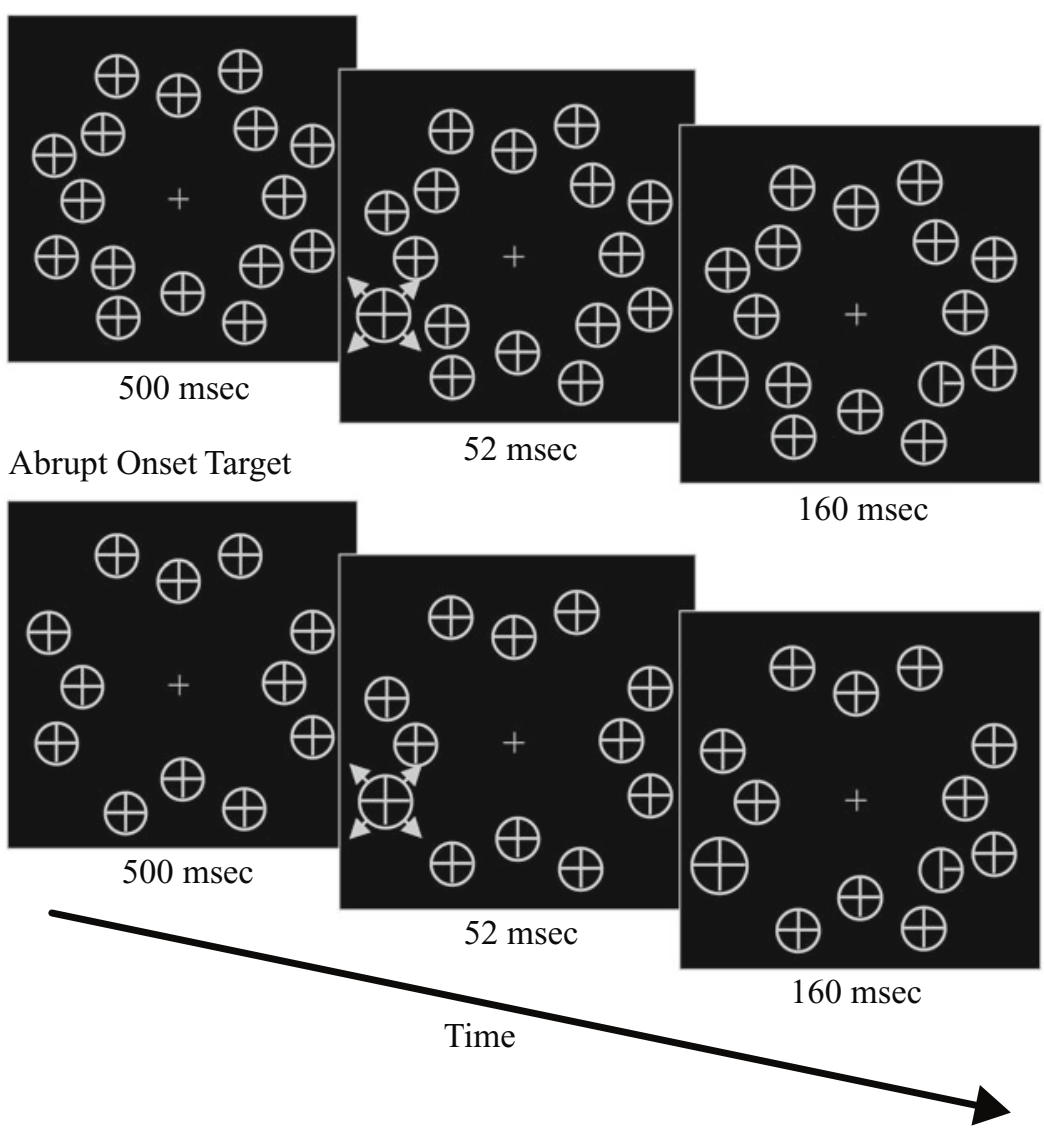

Figure 1. Example stimulus display sequences for the two target types (top, unmasked targets; bottom, abrupt onset targets) in Experiment 1. Each trial sequence began with a preview display of $500 \mathrm{msec}$. Following this preview, one of the distractor items on the outer ring underwent a small $(4 \%)$ or large $(12 \%)$ increase in diameter. After $52 \mathrm{msec}$, the target item was revealed or had its onset on the inner ring and remained for $160 \mathrm{msec}$, followed by a blank screen. Subjects made a speeded response concerning the orientation of the $T$ contained within the target circle. 
ways presented at a location on the inner ring, whereas the distractor item (when presented) was on the outer ring.

Conditions and Procedure. Four conditions were created by crossing two types of targets (onset vs. unmasked) and two types of distractors (small vs. large increase in size). In the small distractor increase conditions, one of the items in the outer ring underwent a $4 \%$ increase in diameter. In the large distractor increase conditions, one of the items in the outer ring increased in diameter by $12 \%$.

The subjects completed 20 practice trials followed by 576 experimental trials divided into 12 blocks of 48 . This total included 144 trials for each combination of target type and distractor size. For each of the four target type/distractor size combinations, the two possible target values were presented an equal number of times at each of the eight possible target locations (yielding four different target-distractor separations). For each target location, the size change distractor appeared an equal number of times at each of its eight possible locations and was absent (control condition) on an additional 16 trials. The ordering of conditions as well as target and distractor positions were randomly mixed within blocks. The subjects were instructed to maintain fixation on the central cross throughout the trial. Incorrect responses were signaled by a computer beep. Speed and accuracy feedback was provided every 48 trials. Experimental sessions lasted approximately $40 \mathrm{~min}$.

\section{Results}

The data from 1 subject were excluded from the analysis because the error rate exceeded $15 \%$. Trials were separated according to the four target type-distractor type combinations as well as the four target-distractor separations (plus the control condition). Outliers in the RT data were trimmed by excluding trials in which responses were faster than $300 \mathrm{msec}$ or more than three standard deviations above the mean RT for a given subject in a given condition. This led to the rejection of fewer than $2 \%$ of the trials within both this and the following experiment. RTs from incorrect trials (representing 9\% of total trials) were excluded from the analysis. In both experiments, the pattern of error rates mimicked that of the RT data, and error rates were not analyzed further.

A comparison of control conditions revealed that onset targets were responded to more quickly than unmasked targets [474 vs. $513 \mathrm{msec} ; t(21)=6.01, p<.001$ ], suggesting that the target type manipulation was effective in altering target salience. Figure 2 shows mean difference RTs (relative to RTs in control conditions) for the four target-distractor combinations across each of the four target-distractor separations. A repeated measures ANOVA with the factors of target type (onset vs. unmasked), distractor type (large change vs. small change), and target-distractor separation was run on the difference RTs. The ANOVA revealed a main effect of target type $[F(1,21)=18.37, p<.001]$, a main effect of distractor type $[F(1,21)=12.16, p<.005]$, and a main effect of target-distractor separation $[F(3,63)=4.45, p<.01]$. Both the target type $\times$ separation $[F(3,63)=5.11, p<$ $.005]$ and the distractor type $\times$ separation $[F(3,63)=$ $3.32, p<.05]$ two-way interactions were statistically significant. The target type $\times$ distractor type two-way interaction did not approach significance $[F(1,21)<1]$, nor did the three-way interaction $[F(3,63)=1.20$, n.s. $]$.

Separate two-way ANOVAs were performed on the trials in which the target was unmasked and on those in

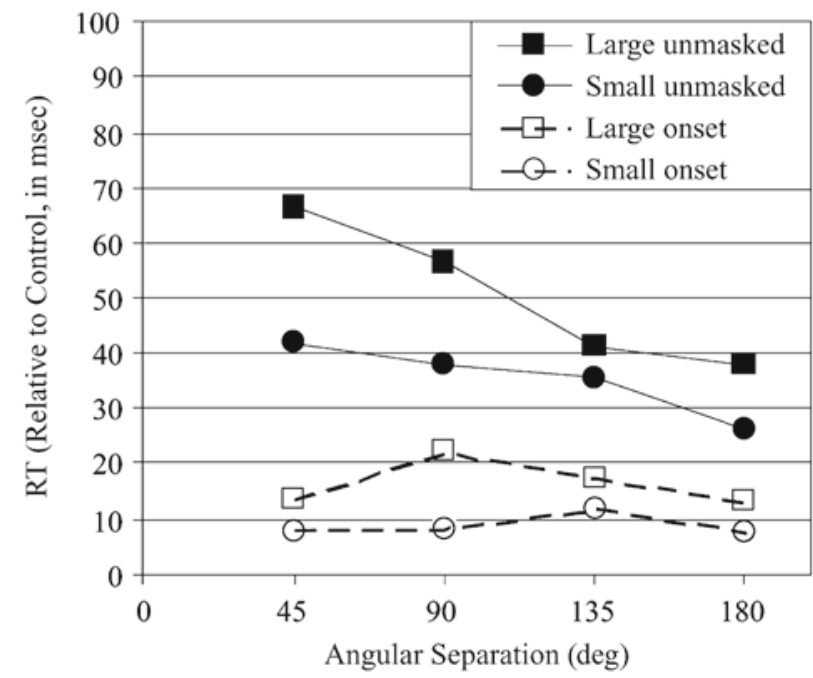

Figure 2. Mean reaction times (relative to the respective control conditions) from Experiment 1 for each target-distractor combination as a function of target-distractor separation. Solid lines and symbols correspond to unmasked targets, and dashed lines and open symbols correspond to onset targets. Small distractor size changes are indicated by circles, and large distractor size changes are indicated by squares.

which the target had an abrupt onset, with distractor type and separation serving as repeated measures factors. For the unmasked targets, there was a main effect of distractor $[F(1,21)=8.31, p<.01]$ and a main effect of separation $[F(3,63)=5.48, p<.05]$, and the interaction between separation and distractor type approached statistical significance $[F(3,63)=2.62, p<.06]$. For the onset targets, neither main effect approached significance [distractor, $F(1,21)=2.11$; separation, $F(3,63)=1.54$ ], nor did the interaction between distractor type and separation $[F(3,63)=1.00]$.

In order to determine whether LAI was observed for each of the possible target type-distractor type combinations, planned linear contrasts for target-distractor separation effects were performed. For the unmasked targets, both the small distractors $[F(1,21)=7.19, p<.05]$ and the large distractors $[F(1,21)=9.95, p<.005]$ were reliable. For the onset targets, neither linear contrast was reliable (both $F_{\mathrm{S}}<1$ ).

\section{Discussion}

The results suggest that both salience manipulations were effective. Responses to onset targets were faster than those to targets that were unmasked, supporting the contention that onset targets were more salient than unmasked targets. In general, interference effects were greater for large distractor changes than for small distractor changes, suggesting that large changes in distractor size were more salient. Moreover, these salience manipulations yielded effects on LAI consistent with the predictions of the salience-based competition models described above. Target discriminations were slowed near size change distractors, and the amount of this slowing depended on the 
magnitude of the size change. This LAI is presumed to reflect a competition for representation by shared neural resources. As the distractor becomes more attentionally salient, it gains control over a larger proportion of these shared resources, leaving fewer for target representation. However, this occurred only for unmasked targets. Onset targets, presumably possessing greater salience, appeared to be relatively immune from interference from the size change distractors. In terms of a salience-based competition explanation, these onset targets were able to win the competition for representation with both types of size distractors. ${ }^{3}$

\section{EXPERIMENT 2}

Experiment 1 revealed that stimulus-based manipulations of salience affected the magnitude of interference between a distractor and the target item. In Experiment 2, the salience of a distractor item was manipulated using a probability manipulation as opposed to the stimulus manipulation used in Experiment 1. In the present experiment, the salient distractor was a color singleton embedded among the other distractor items. For half of the subjects, the color singleton was predictive of the target location (i.e., the target was the singleton on $50 \%$ of the trials). This should bestow a relatively high attentional salience on the color singleton. For the other half of the subjects, the color singleton was unpredictive of the location of the target, yielding a situation in which the color singleton should have lower attentional salience (relative to the case in the predictive singleton condition). Across the two conditions, the stimulus displays used were physically identical, differing only in their relative frequency.

\section{Method}

Subjects. Fifty-six students from the State University of New York at Geneseo participated in the experiment in exchange for course credit. All of the subjects reported normal or corrected-tonormal visual acuity and were screened for color blindness using Ishihara color plates.

Apparatus and Stimuli. The apparatus was identical to that used in Experiment 1. Stimulus items were again circles with crosses in the middle and were the same size as those used in Experiment 1. The stimuli were either red (CIE $x=.54, y=.29$; luminance $=2.40 \mathrm{~cd} /$ $\left.\mathrm{m}^{2}\right)$ or green (CIE $x=.31, y=.51$; luminance $\left.=2.56 \mathrm{~cd} / \mathrm{m}^{2}\right)$ and were presented on a black background (luminance $=0.28 \mathrm{~cd} / \mathrm{m}^{2}$ ). A single ring of stimulus circles was presented along an imaginary ring with a radius of $4.5^{\circ}$. Figure 3 shows the frame sequence, which included an 82-msec preview frame followed by a 146-msec target frame. As in Experiment 1, a target item was created by removing the left or the right half of the horizontal portion of a cross (yielding a $\mathrm{T}$ with the stem pointing left or right). The subjects made a speeded discrimination response regarding the shape of the target (stem to the left or to the right).

Conditions and Procedure. Half of the subjects were randomly assigned to the unpredictive singleton condition. These subjects completed a session that included 20 practice trials followed by 9 blocks of 48 experimental trials, for a total of 432 experimental trials. For half of these subjects, a green singleton among red items was used, whereas the other half of the subjects experienced a red singleton among green items. The target item was presented equally often at each of the eight possible locations. The color singleton

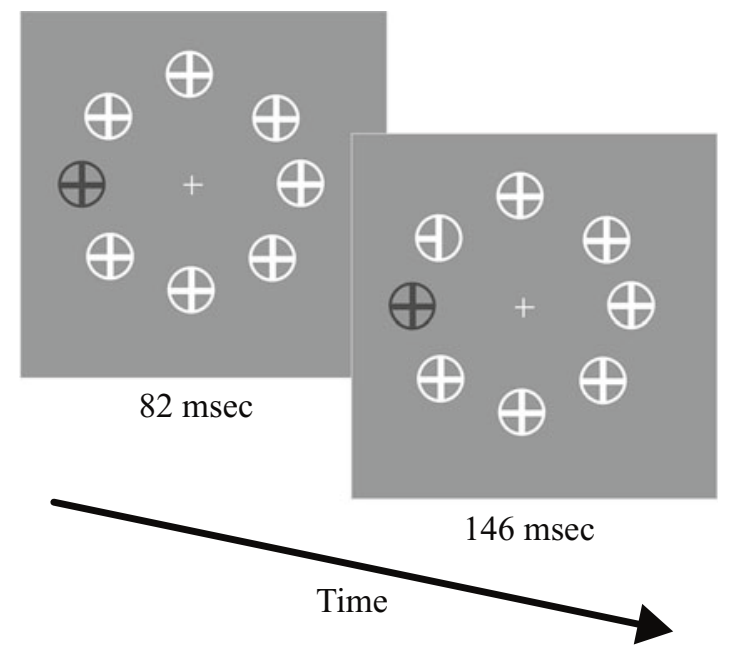

Figure 3. Example stimulus display sequence for Experiment 2. A preview display containing a color singleton was initially presented. After $82 \mathrm{msec}$, one of the stimulus items lost a line segment, revealing the target's location and identity. Subjects made a speeded response concerning the orientation of the $T$ contained within the target circle.

distractor also appeared equally often at each of the eight locations, and its location was uncorrelated with the location of the target. The color singleton was withheld on 48 of the trials to yield a control condition.

The other half of the subjects were assigned to the predictive singleton condition. These subjects completed a session that included 20 practice trials followed by 10 blocks of 48 experimental trials, for a total of 480 experimental trials. Half of the subjects saw displays containing a green singleton among red items, and the other half viewed displays with a red singleton among green items. The target item appeared equally often at each of the eight target locations. The color singleton was withheld on 32 control trials and was present in the remaining 448 trials. For half of these 448 trials, the target appeared at the color singleton location. For the other 224 such trials, the color singleton was presented equally often at one of the seven nontarget locations.

For both tasks, the subjects were instructed to maintain fixation on the central cross throughout the trial. Incorrect responses were signaled by a computer beep. Speed and accuracy feedback was provided after every 48 trials. Each session lasted approximately $30 \mathrm{~min}$.

\section{Results}

Four subjects in the unpredictive singleton condition and 5 subjects in the predictive singleton condition were replaced because their error rates exceeded $15 \%$. RTs were trimmed for outliers ( $<2 \%$ for each condition) as in Experiment 1. Figure 4 shows the mean correct RTs (relative to the control condition) for the two probability conditions as a function of target-distractor separation. RTs to color singleton targets in the predictive singleton condition were reliably shorter than those to targets in the corresponding control condition $[t(27)=6.31, p<$ $.001]$, whereas RTs to color singletons in the unpredictive singleton condition did not differ from those in the corresponding control condition $[t(27)=-.24$, n.s.]. A mixed-model ANOVA, with the between-subjects factor 


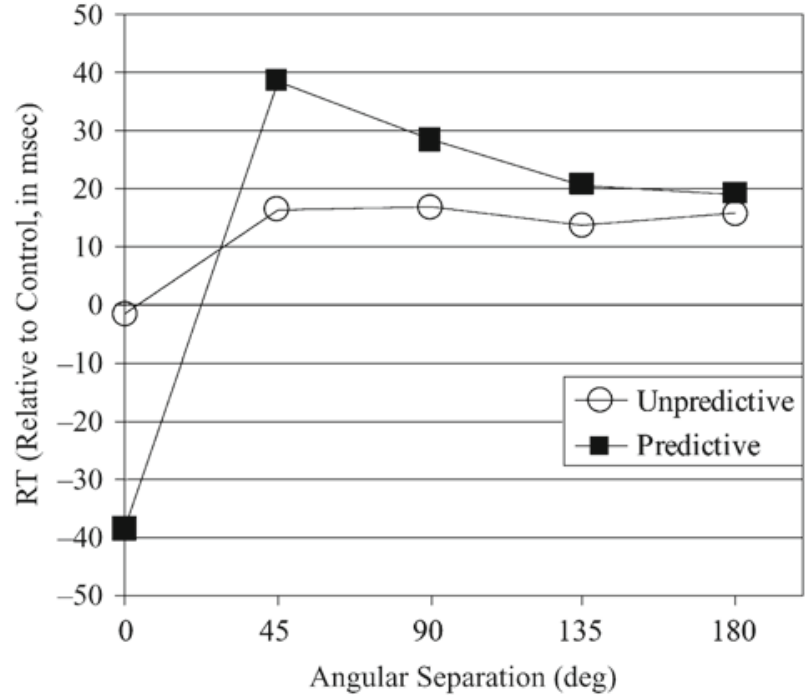

Figure 4. Mean reaction times (relative to the control condition) from Experiment 2 for the predictive and unpredictive conditions as a function of target-distractor separation. A separation of 0 corresponds to trials in which the target appeared at the color singleton location.

of condition (predictive vs. unpredictive singleton) and the repeated measures factor of target-distractor separation (using the four nontarget locations), was conducted on the difference RTs (relative to the respective control conditions). The ANOVA revealed no main effect of condition type $[F(1,54)=1.10$, n.s. $]$, a main effect of separation $[F(3,162)=4.64, p<.005]$, and a significant interaction between condition and separation $[F(3,162)=3.35$, $p<.05]$. This interaction was followed up with the use of linear contrasts on the factor of separation separately for each condition. The linear contrast for the unpredictive singleton condition was not reliable $[F(3,81)<1]$, whereas the linear contrast for separation in the predictive singleton condition was statistically significant $[F(3,81)=5.08, p<.005]$.

\section{Discussion}

The results suggest that the probability manipulation was effective in controlling the attentional salience of the color singleton. In the predictive singleton condition but not in the unpredictive singleton condition, responses to color singleton targets were reliably faster than those in the control condition, suggesting that the color singleton captured attention in the predictive but not in the unpredictive singleton condition. The effects of the attentional salience of the color singleton were also manifest in the responses to targets at the nonsingleton locations, with singleton-target separation effects (i.e., LAIs) being observed in the predictive but not in the unpredictive singleton condition. This result complements the results of Experiment 1 , demonstrating that the top-down attentional salience of a distractor item mediates the magnitude of LAI observed.
The lack of LAI in the unpredictive singleton condition was slightly surprising, since previous studies have revealed LAI surrounding an uninformative color singleton (Mounts, 2000a; Theeuwes \& Godijn, 2001). There are several possible explanations for this discrepancy. First, this may reflect differences between the target stimuli used in these studies. In both Mounts (2000a) and Theeuwes and Godijn (2001), the target was a shape singleton, whereas in the present experiment the targets were not only shape singletons but also transient events (caused by the offset of one of the line segments). If subjects used the transient cue to locate the target in this task, then the contingent attentional capture hypothesis (Folk, Remington, \& Johnston, 1992; Folk, Remington, \& Wright, 1994) would predict that an unpredictive static color singleton would not capture attention. Second, the features defining the target and the distractor were held constant for each subject. Theeuwes and Burger (1998) found that interference from a singleton distractor could be avoided under such conditions. A final possible explanation derives from the findings of Experiment 1, which showed that if the target is high enough in salience, LAI (and perhaps attentional capture by a less salient distractor) can be avoided. Thus, perhaps the salience of the offset transient was great enough to prevent capture by the color singleton in the unpredictive singleton condition but not in the predictive singleton condition, in which the attentional salience of the color singleton was higher. Finally, even though attentional capture was not evident in the unpredictive singleton condition, responses at nonsingleton locations were reliably slowed relative to control. This may reflect a singleton filtering cost, as postulated by Folk and Remington (1998).

\section{GENERAL DISCUSSION}

The results of two experiments demonstrated the role of target and distractor salience in mediating LAI. In both experiments, LAI was accentuated with increases to the attentional salience of the distractor. In Experiment 1, the presence of LAI depended on the salience of the target as well. These results are consistent with competitionbased models of attentional selection (see, e.g., Cave, 2001; Deco \& Rolls, 2004; Desimone \& Duncan, 1995; Tsotsos et al., 1995). According to this general class of models, performance to a target depends on the processing resources that the target is able to secure through salience-based competitions with other items in the visual scene. LAI is observed when spatially proximal targets and attentionally salient distractors compete for processing resources, with target identification performance reflecting the proportion of resources available to represent the target. Experiment 1 demonstrated that the ability to represent the target depends on both the salience of the target and the salience of the distractor. Moreover, Experiment 2 demonstrated that this competition can be mediated solely through the top-down control of the attentional salience of a distractor, since different patterns of LAI 
were obtained with identical stimulus displays across the predictive and unpredictive singleton conditions. These results, obtained in a search-style task, conceptually replicate the findings of Mounts and Gavett (2004) and are consistent with models of attentional selection based on competitive interactions.

Experiment 2, in which LAI can be manipulated via attentional set, suggests that such a manipulation cannot be accounted for by simple sensory masking (cf. Efron \& Yund, 1999), since retinal input was equated across the predictive and unpredictive singleton conditions. Other studies in the literature have also ruled out simple sensory masking accounts of LAI (e.g., Bahcall \& Kowler, 1999, Experiments 2 and 3; McCarley et al., 2004; Mounts \& Gavett, 2004, Experiment 4). Nonetheless, the mechanisms underlying LAI may be related to those that produce masking phenomena such as crowding (see, e.g., Bouma, 1970; Levi, Klein, \& Aitsebaomo, 1985; Toet \& Levi, 1992; Wolford \& Chambers, 1983). Intriligator and Cavanagh (2001) observed that attentional resolution was limited in the visual periphery and decreased with increased retinal eccentricity. They argued (along with He, Cavanagh, \& Intriligator, 1996) that phenomena such as crowding might result from poor attentional resolution (concluding that attentional resolution was coarser than visual resolution at any given eccentricity). In the crowding paradigm, as the spacing between the target and flanking items is decreased, limits in attentional resolution result in difficulty in extracting information from the target location only. The target representation is therefore degraded by feature information coming from flanker locations, which ultimately renders the target unrecognizable. Intriligator and Cavanagh also point out that in the typical crowding task the target is defined solely by its location. When additional features (e.g., a unique color or orientation; see Kooi, Toet, Tripathy, \& Levi, 1994) are available to define the target, crowding is reduced. Intriligator and Cavanagh argue that these additional cues aid in the attentional selection of the target, thereby reducing interference from flanker features.

In studies of LAI, uniformly dense displays are typically employed by using "filler distractors," so that the target is always flanked by similar items across the various target-salient distractor separations. However, the target is also typically specified by some additional cue (be it a location precue or a unique feature) that renders it discernible from the flanking distractors, as is the salient distractor. ${ }^{4}$ With distraction effects from the filler distractors equated across the separation conditions, target identification performance across conditions will be sensitive to the separation between the target and the salient distractor when the stimulus or the attentional salience of the second item makes it difficult or impossible to filter to the same extent as the filler distractors.

Thus, LAI may be a special case of the crowding phenomenon, wherein one of the flanks has enhanced "crowding" capabilities due to increased stimulus or attentional salience. Future research is necessary to examine whether
LAI exhibits characteristics similar to those of crowding. Existing research suggests that this may be the case. Bahcall and Kowler (1999) and Cutzu and Tsotsos (2003) found that the spatial extent of LAI varied with retinal eccentricity, as does crowding (see, e.g., Toet \& Levi, 1992). Furthermore, preliminary data from this laboratory suggest that salient distractors more eccentric than the targets produce larger regions of LAI than do salient distractors located more centrally, as is observed in crowding (see, e.g., Banks, Larson, \& Prinzmetal, 1979). It will also be informative to determine the stage processing affected by LAI, since crowding has been postulated to affect the feature integration stage (see, e.g., Pelli, Palomares, \& Majaj, 2004).

In addition to evaluating the relationship between LAI and crowding, determining the stage of processing affected by LAI will allow a further evaluation of the competitive interaction models described above to account for this phenomenon. According to these models, resource limitations affect the perceptual representation of the target. However, alternative models of visual processing postulate that attentional effects such as cuing can be modeled solely at the decision level, with attention having no effect on the perceptual representation of the stimuli (see, e.g., Eckstein, Shimozaki, \& Abbey, 2002). Using a Posner-style cuing procedure (see, e.g., Posner, 1980), Eckstein et al. (2002) found no evidence that attention causes changes in perceptual filters and concluded that attentional cuing effects could be accounted for solely by decisional processes. Specifically, Eckstein et al.'s (2002) model assumed that in the decision stage the weighting of information from the cued location is enhanced whereas the weighting at the uncued location was reduced. Such a shift in weights was sufficient to capture cue validity effects in the absence of any changes to the underlying perceptual representations. Target facilitation in the predictive condition of Experiment 2 of the present work might similarly be accounted for by such a decisional weighting model. However, in order to account for the observed separation effects when the color singleton was not the target, a decisional weighting model would have to propose that spatial constraints regulate information flow into the decisional stage, in such a way that decisional weightings at noncued locations would vary with distance from the cue (in this case, the color singleton).

More generally, the success of recent signal detection models of search performance (e.g., Eckstein, Thomas, Palmer, \& Shimozaki, 2000; Palmer, 1994; Palmer, Ames, \& Lindsey, 1993; Palmer, Verghese, \& Pavel, 2000) has brought into question the need for limited-capacity processing assumed in many models of visual attention. ${ }^{5}$ For example, Palmer et al. (2000) showed that visual search phenomena such as set size effects and the effects of distractor heterogeneity could be accounted for successfully by models based on signal detection theory. These models assume independence of items in the stimulus display (i.e., items are processed in parallel and with unlimited capacity). In contrast to this assumption, in both the at- 
tentional capture tasks employed in the present study and divided attention tasks (i.e., dual-identification and samedifferent tasks), the observation of LAI appears to impose spatial limits on the ability of attentional processes to operate on items independently (see also McCarley, Mounts, \& Kramer, 2005). This suggests that either (1) the perceptual representations of spatially proximal stimuli are degraded due to local capacity limitations (as proposed by the competitive interaction models) or (2) spatial constraints exist regarding the attentional regulation of information flow into the decisional stage. The latter alternative would be consistent with observed differences in the spatial resolution of visual versus attentional processing (Intriligator \& Cavanagh, 2001). Research to determine the stage(s) of processing affected by spatially mediated interference will aid in discriminating between statistical decision models and capacity-limited perceptual models as explanations of the phenomenon. Of course, it should be noted that the competitive interaction models of attentional selection and the signal detection models of search performance need not be mutually exclusive; Verghese (2001) illustrates how attentionally modulated competitive interactions among stimuli could be modeled within a signal detection theory approach, correctly predicting the effect of an attentional cue on search performance.

\section{REFERENCES}

Bahcall, D. O., \& Kowler, E. (1999). Attentional interference at small spatial separations. Vision Research, 39, 71-86.

Banks, W. P., Larson, D. W., \& Prinzmetal, W. (1979). Asymmetry of visual interference. Perception \& Psychophysics, 25, 447-456.

Bouma, H. (1970). Interaction effects in parafoveal letter recognition. Nature, 226, 177-178.

Caputo, G., \& Guerra, S. (1998). Attentional selection by distractor inhibition. Vision Research, 38, 669-689.

CAVE, K. R. (2001). Selection can be performed effectively without temporal binding, but could be even more effective with it. Visual Cognition, 8, 467-487.

Cutzu, F., \& Tsotsos, J. K. (2003). The selective tuning model of attention: Psychophysical evidence for a suppressive annulus around an attended item. Vision Research, 43, 205-219.

Deco, G., \& Rolls, E. T. (2004). A neurodynamical cortical model of visual attention and invariant object recognition. Vision Research, 44, 621-642.

Desimone, R. (1998). Visual attention mediated by biased competition in extrastriate visual cortex. Philosophical Transactions of the Royal Society of London, 353, 1245-1255.

Desimone, R., \& Duncan, J. (1995). Neural mechanisms of selective visual attention. Annual Review of Neuroscience, 18, 193-222.

Eckstein, M. P., Shimozaki, S. S., \& Abbey, C. K. (2002). The footprints of visual attention in the Posner cueing paradigm revealed by classification images. Journal of Vision, 2, 25-45.

Eckstein, M. P., Thomas, J. P., Palmer, J., \& Shimozaki, S. S. (2000). A signal detection model predicts the effects of set size on visual search accuracy for feature, conjunction, triple conjunction, and disjunction displays. Perception \& Psychophysics, 62, 425-451.

EFron, R., \& Yund, E. W. (1999). Attentional inhibition or paracontrast? Brain \& Cognition, 41, 111-149.

Folk, C. L., \& Remington, R. W. (1998). Selectivity in distraction by irrelevant featural singletons: Evidence for two forms of attentional capture. Journal of Experimental Psychology: Human Perception \& Performance, 24, 847-858.

Folk, C. L., Remington, R. W., \& Johnston, J. C. (1992). Involuntary covert orienting is contingent on attentional control settings. Journal of Experimental Psychology: Human Perception \& Performance, 18, 1030-1044.

Folk, C. L., Remington, R. W., \& Wright, J. H. (1994). The structure of attentional control: Contingent attentional capture by apparent motion, abrupt onset, and color. Journal of Experimental Psychology: Human Perception \& Performance, 20, 317-329.

Franconeri, S. L., \& Simons, D. J. (2003). Moving and looming stimuli capture attention. Perception \& Psychophysics, 65, 999-1010.

He, S., Cavanagh, P., \& Intriligator, J. (1996). Attentional resolution and the locus of visual awareness. Nature, 383, 334-337.

INTRILIGATOR, J., \& CAVANAGH, P. (2001). The spatial resolution of visual attention. Cognitive Psychology, 43, 171-216.

Kastner, S., De WeErd, P., Pinsk, M. A., Elizondo, M. I., Desimone, R., \& UNGERLEIDER, L. G. (2001). Modulation of sensory suppression: Implications for receptive field sizes in the human visual cortex. Journal of Neurophysiology, 86, 1398-1411.

Keysers, C., \& PerretT, D. I. (2002). Visual masking and RSVP reveal neural competition. Trends in Cognitive Sciences, 6, 120-125.

Kooi, F. L., Toet, A., Tripathy, S. P., \& LeVI, D. M. (1994). The effect of similarity and duration on spatial interaction in peripheral vision. Spatial Vision, 8, 255-279.

LeVi, D. M., Klein, S. A., \& Aitsebaomo, A. P. (1985). Vernier acuity, crowding and cortical magnification. Vision Research, 25, 963-977.

McCarley, J. S., Mounts, J. R. W., \& Kramer, A. F. (2004). Agerelated differences in localized attentional interference. Psychology \& Aging, 19, 203-210.

McCarley, J. S., Mounts, J. R. W., \& Kramer, A. F. (2005). Spatially-mediated capacity limits in attentive visual perception. Manuscript submitted for publication.

Mounts, J. R. W. (2000a). Attentional capture by abrupt onsets and feature singletons produces inhibitory surrounds. Perception \& Psychophysics, 62, 1485-1493.

MounTs, J. R. W. (2000b). Evidence for suppressive mechanisms in attentional selection: Feature singletons produce inhibitory surrounds. Perception \& Psychophysics, 62, 969-983.

Mounts, J. R. W., \& GavetT, B. E. (2004). The role of salience in localized attentional interference. Vision Research, 44, 1575-1588.

Mounts, J. R. W., \& Tomaselli, R. G. (2005). Competition for representation is mediated by relative attentional salience. Acta Psychologica, 118, 261-275.

PAlmer, J. (1994). Set-size effects in visual search: The effect of attention is independent of the stimulus for simple tasks. Vision Research, 34, 1703-1721.

Palmer, J., Ames, C. T., \& Lindsey, D. T. (1993). Measuring the effect of attention on simple visual search. Journal of Experimental Psychology: Human Perception \& Performance, 19, 108-130.

Palmer, J., Verghese, P., \& Pavel, M. (2000). The psychophysics of visual search. Vision Research, 40, 1227-1268.

Pelli, D. G., Palomares, M., \& Majaj, N. J. (2004). Crowding is unlike ordinary masking: Distinguishing feature integration from detection. Journal of Vision, 4, 1136-1169.

Posner, M. I. (1980). Orienting of attention. Quarterly Journal of Experimental Psychology, 32, 3-25.

Reynolds, J. H., Chelazzi, L., \& Desimone, R. (1999). Competitive mechanisms subserve attention in macaque areas V2 and V4. Journal of Neuroscience, 19, 1736-1753.

Reynolds, J. H., \& Desimone, R. (2003). Interacting roles of attention and visual salience in V4. Neuron, 37, 853-863.

Sperling, G., \& Dosher, B. (1986). Strategy and optimization in human information processing. In K. R. Boff, L. Kaufman, \& J. P. Thomas (Eds.), Handbook of perception and human performance (pp. 2-1 to 2-65). New York: Wiley.

TheEuwes, J., \& Burger, R. (1998). Attentional control during visual search: The effect of irrelevant singletons. Journal of Experimental Psychology: Human Perception \& Performance, 24, 1342-1353.

Theeuwes, J., \& GodiJn, R. (2001). Attentional and oculomotor capture. In C. Folk \& B. Gibson (Eds.), Attraction, distraction, and action (pp. 121-149). New York: Elsevier.

ToET, A., \& LEVI, D. M. (1992). The two-dimensional shape of spatial interaction zones in the parafovea. Vision Research, 32, 1349-1357.

Tsotsos, J. K., Culhane, S. M., WaI, W. Y. K., Lai, Y., Davis, N., \& 
NufLo, F. (1995). Modeling visual attention via selective tuning. $A r$ tificial Intelligence, 78, 507-545.

Turatto, M., \& Galfano, G. (2001). Attentional capture by color without any relevant attentional set. Perception \& Psychophysics, 63 , 286-297.

Verghese, P. (2001). Visual search and attention: A signal detection theory approach. Neuron, 31, 523-535.

Wolford, G., \& Chambers, L. (1983). Lateral masking as a function of spacing. Perception \& Psychophysics, 33, 129-138.

Yantis, S. (1996). Attentional capture in vision. In A. F. Kramer, M. G. H. Coles, \& G. D. Logan (Eds.), Converging operations in the study of visual selective attention (pp. 45-76). Washington, DC: American Psychological Association.

YAnTis, S., \& Hillstrom, A. P. (1994). Stimulus-driven attentional capture: Evidence from equiluminant visual objects. Journal of Experimental Psychology: Human Perception \& Performance, 20, 95-107.

YANTIS, S., \& JonIDES, J. (1984). Abrupt visual onsets and selective attention: Evidence from visual search. Journal of Experimental Psychology: Human Perception \& Performance, 10, 601-621.

YANTIS, S., \& Jonides, J. (1990). Abrupt visual onsets and selective attention: Voluntary versus automatic allocation. Journal of Experimental Psychology: Human Perception \& Performance, 16, 121-134.

\section{NOTES}

1. The term localized attentional interference (LAI) will be used even when the competition is rooted in stimulus-driven salience. This is because the objects are presumed to be competing for attentional selection, which in these models corresponds to an enhanced neural representation. Moreover, Mounts and Gavett (2004) found that both attentionbased and stimulus-driven salience yielded similar interference effects, consistent with single-cell data (Reynolds \& Desimone, 2003).
2. The use of the term salience here encompasses both stimulus-driven and attentionally mediated salience. Although the neural substrates underlying the attentional priority given to looming stimuli is poorly understood, the behavioral evidence suggests that such size changes do enhance the attentional salience of such stimuli (see, e.g., Franconeri \& Simons, 2003; Mounts \& Gavett, 2004). Moreover, attentionally mediated and stimulus-mediated manipulations appear to have comparable effects, both at the behavioral (see, e.g., Mounts \& Gavett, 2004) and the single-cell (see, e.g., Reynolds \& Desimone, 2003) levels.

3 . One might argue that the immunity of the onset targets from interference arises because of the source of their salience (i.e., stimulus driven). For instance, one could imagine a system in which items with high attentional priority due to stimulus factors (e.g., abrupt onsets) always take priority over items with high attentional priority due to attentional settings. However, Yantis and Jonides (1990) found that spatially cuing a subsequent (offset) target (an attentional manipulation) rendered it immune from the distracting effects of an abrupt onset distractor.

4. The example given here is for attentional capture tasks involving a target and an attentionally salient distractor. However, the same is true of other types of tasks that yield LAI, such as dual-identification tasks and same-different tasks.

5. The type of decision models described in this section have typically been applied to simple, single-dimension detection tasks (e.g., detection of a luminance increment or a hue difference). The task used in the present study (shape identification) may involve different information integration and decision processes. Nonetheless, it seems reasonable that such models can be successfully extended to the present type of stimulus decision (see, e.g., Palmer et al., 2000).

(Manuscript received December 5, 2003; revision accepted for publication February 11, 2005.) 